\title{
Patent foramen ovale in patients with tension headache: is it as common as in migraineurs? An age- and sex-matched comparative study
}

\author{
Ali Reza Moaref - Peyman Petramfar - Kamran Aghasadeghi · \\ Mahmood Zamirian - Mohammad Bagher Sharifkazemi · \\ Shahed Rezaian · Sasan Afifi · Najaf Zare · Gholam Reza Rezaian
}

Received: 11 May 2009/Accepted: 24 August 2009/Published online: 12 September 2009

(C) Springer-Verlag 2009

\begin{abstract}
The association of patent foramen ovale (PFO) and atrial septal aneurysm (ASA) with migraine headache attack (MHA) has been clearly shown. The same findings have been recently demonstrated also in cluster headache. Although tension-type headaches (TTH) are the most common kind of headache, their association with these atrial septal abnormalities has never been studied before. The study was conducted to clarify whether there was a significant association between the presence of such atrial septal abnormalities and tension headache, when compared with migraineurs. One hundred consecutive patients with migraine and 100 age- and sex-matched subjects with TTH and 50 healthy volunteers with no headache were enrolled in the study and underwent a complete transesophageal echocardiographic study with contrast injections at rest and with the Valsalva maneuver. There was
\end{abstract}

\footnotetext{
A. R. Moaref · K. Aghasadeghi · M. Zamirian ·

M. B. Sharifkazemi · G. R. Rezaian $(\bowtie)$

Department of Medicine (Cardiology), Namazee Hospital,

Shiraz University of Medical Sciences,

71937-11351 Shiraz, Iran

e-mail: grezaian@gmail.com

A. R. Moaref

e-mail: moarefa@sums.ac.ir

K. Aghasadeghi

e-mail: aghasadeghk@sums.ac.ir

M. Zamirian

e-mail: drzamirian@yahoo.com

M. B. Sharifkazemi

e-mail: msharifkazemi@yahoo.com

P. Petramfar

Department of Medicine (Neurology),

Shiraz University of Medical Sciences, Shiraz, Iran

e-mail: petramfar@yahoo.com
}

no significant difference between the age and the sex of the participants of the three groups. The overall prevalence of PFO was $23 \%$ in patients with TTH and that of large PFOs was only $11 \%$. The $23 \%$ prevalence of PFO in patients with TTH was not statistically different from $16 \%$ found in our normal control group. Furthermore, we found a significantly higher prevalence of PFO in migraineurs $(50 \%)$ when compared with patients with tension headache $(p<0.001)$. This was also true for the collective presence of large PFOs and ASAs $(35 \%)(p<0.001)$. Although atrial septal anomalies have an association with MHA, they do not have a significant association with TTH.

Keywords Tension-type headache $\cdot$ Migraine headache . Patent foramen ovale $\cdot$ Atrial septal aneurysm

\author{
S. Rezaian \\ Alzahra Cardiovascular Research Center, \\ Shiraz University of Medical Sciences, Shiraz, Iran \\ e-mail: shahedrezaian@gmail.com \\ S. Afifi \\ Department of Cardiology, \\ Shiraz University of Medical Sciences, Shiraz, Iran \\ e-mail: sasanafifi@yahoo.com \\ N. Zare \\ Department of Biostatistics, \\ Shiraz University of Medical Sciences, Shiraz, Iran \\ e-mail: najafzare@sums.ac.ir
}




\section{Introduction}

Several studies have shown an association between patent foramen ovale (PFO) and migraine headache attack (MHA), and that PFO is more common in migraineurs with aura [1]. In addition, PFO and atrial septal aneurysm (ASA) have been found to be an important risk factor for stroke [2]. A similar association has recently been shown in patients with cluster headache $(\mathrm{CH})$ [3-5] raising the possibility that such atrial septal abnormalities might play a role not only for migraine, but also for other types of headache as well. Although tensiontype headaches (TTH) are the most common kind of headache [6], their association with atrial septal abnormalities has never been studied before. If these structural atrial septal abnormalities are predisposing conditions for different headache disorders, then they should be also commonly found in subjects with TTH or be at least as common as in patients with MHA. This study, therefore, was designed to find out the frequency and the association of atrial septal abnormalities specifically PFOs and large PFOs with TTH and compare them with the migraineurs and a group of normal subjects.

\section{Materials and methods}

\section{Inclusion and exclusion criteria}

Patients referring to our university hospital neurology headache clinic were recruited. Only subjects aged 15 years and older with headaches fulfilling the International Headache Society Criteria for MHA (with or without aura) or TTH, could properly answer the questions and accepted having transesophageal echocardiography (TEE) were included. Younger subjects, patients with medication overuse and those not tolerating TEE or having insufficient echocardiographic data (poor visualization of cardiac chambers and/or the atrial septum) were excluded.

To exclude drug overuse, patients were asked for:

1. The type (NSAIDS, triptans, tricyclic antidepressants, ergotamine, etc.), frequency and duration of the drugs used for acute and/or symptomatic treatment of their headaches and,

2. If their headache has had a very close temporal relation to the use of their medications.

3. If there has been a marked aggravation of their preexisting headache.

4. If they have definitely noted worsening of their primary headache by the use of a specific drug.

All suspected cases were asked to stop the medication(s) for 2 months and were reassessed afterwards for improvement or resolution of their headache. Subjects were included only after the exclusion of drug overuse headache.
The control subjects were included only when they had neither migraine, TTH nor any other headache for at least the past 6 months.

\section{Patients and controls}

From May 2007 to April 2009, 1,164 patients and 132 normal volunteers with an age range of 15-60 years were visited. Information about migraine and tension headache was obtained by a neurologist via a face-to-face interview using a structured questionnaire. Drug overuse headache was proved to be present in 674 subjects and 113 patients had other headache types not fitting the study protocol. Of remaining 394 patients who could be enrolled into the study, 191 subjects had chronic TTH and 206 were migraineurs. Following the exclusion of patients with no interest for participation, refused to have TEE and/or could not be age- and sex matched or had other exclusion criteria; 100 patients with migraine and 100 age- and sex-matched subjects with chronic TTH and 50 healthy volunteers with no headache in the last 6 months were enrolled in the study. The control subjects were recruited from the family members and friends of the study participants as well as from those of patients referring to the cardiology clinics. The aim and scope of the study were explained and TEE was demonstrated. All had an interview with our neurologist to ensure fulfilling the study protocol. None of the volunteers were paid for participation.

The diagnosis of the headaches was based on the International Headache Society criteria, second edition [7]. All participants gave a written informed consent and the study protocol was approved by the Institutional Ethics Committee.

\section{Transesophageal echocardiography}

The echocardiographic instrument was Vivid 3 (GE Medical Systems, WI, USA) in all subjects who underwent a complete TEE study including color Doppler using a multiplane 6T, TEE probe.

The TEE study was done with the patient in the left lateral decubitus position. Lidocaine spray was used for topical anesthesia of the pharynx. After optimal visualization of all cardiac chambers and the atrial septum, contrast injections were done in the mid-esophageal short axis and bicaval views in the $40^{\circ}-60^{\circ}$ and $90^{\circ}-110^{\circ}$ image planes of the transducer, respectively.

\section{Contrast medium}

Two $10 \mathrm{ml}$ syringes were used one containing $9 \mathrm{ml}$ of physiologic saline $(0.9 \% \mathrm{NaCl})$ solution and the other containing $1 \mathrm{ml}$ of air. The contents of both syringes were 
rapidly mixed through a three-way stopcock until a homogeneous solution was obtained. It was then rapidly injected into a right antecubital vein through an 18-gauge venous canula, while the patient was at rest. The examination was subsequently repeated with Valsalva maneuver. The overall duration of Valsalva maneuver was $10 \mathrm{~s}$. To ensure a perfect procedure, all patients were trained to perform the maneuver beforehand.

\section{Severity of shunt}

The shunting was considered small when 3-9 micro bubbles appeared in the left atrium (LA) either spontaneously or after Valsalva maneuver and within three cardiac cycles after the complete opacification of the right atrium by the contrast medium [8]. The degree of shunting was defined as medium or large with the presence of 10-30 or $>30$ micro bubbles in the LA, respectively. A more than $4 \mathrm{~mm}$ separation between septum primum and secundum was considered to be indicative of a large PFO.

\section{Statistical analysis}

Statistical analysis was performed using the Statistical Package for Social Sciences (SPSS 15, Chicago, IL, USA). Analysis of variance was used for mean age comparisons and also $\chi^{2}$ test was used as needed. A $p$ value $<0.05$ was considered significant.

\section{Results}

Demographic data of the three groups are shown in Table 1. There was no significant difference between the age and sex of the participants.
Females were predominant in all three groups $(80 \%)$. All patients with chronic TTH had attacks with a frequency of $\geq 4$ times per week. The overall prevalence of PFO was $23 \%$ in patients with TTH and that of large PFOs was only $11 \%$.

In normal subjects, the frequency of PFOs and large PFOs were 16 and $10 \%$, respectively. These frequencies, however, were not significantly higher in patients with TTH compared with the normal subjects (odd's ratio $=1.5,95 \%$ confidence interval $[\mathrm{CI}] 0.64-3.81, p=0.318$ ) and (odd's ratio $=1.11,[\mathrm{CI}] 0.36-3.40, p=0.852)$, respectively.

Aura was detected in $32 \%$ of patients with MHA. The frequency of headache attacks ranged from $\leq 1$ to over 5 headache days per month (once every 2 months to twice per week), leading to activity restriction. Auras were visual in all subjects. The frequency of PFO was $62.5 \%$ in aura-positive patients and $44.1 \%$ in aura-negative subjects $(p=0.086)$, while large PFOs were present in $53.1 \%$ of former group compared with $26.5 \%$ of the latter one $(p=0.009)$.

PFOs and large PFOs were, however, much more prevalent in MHA patients than the normal control group (odd's ratio $=5.25,[\mathrm{CI}] 2.24-12.30, p<0.001$ ) and (odd's ratio $=4.85,[\mathrm{CI}] 1.76-13.32, p=0.001$ ), respectively. These atrial septal anomalies were significantly more common in migraineurs than in TTH group (Table 2).

\section{Discussion}

Tension headache is the most common type of headache [6]. Migraine is also a common neurological disorder with an overall prevalence of about $12 \%$ in the general population [9]; on the other hand, PFO is a common cardiac finding as well. In their large autopsy series of 965 normal hearts Hagen et al. [10] found the prevalence of PFO to be $27 \%$.
Table 1 Demographic data of patients with TTH, MHA and the control group

MHA migraine headache, $n$ number, TTH tension-type headache

Table 2 The prevalence of PFOs and large PFOs/ASAs in TTH patients versus migraineurs

ASA atrial septal aneurysm, $C I$ confidence interval, $M H A$ migraine headache, $n$ number, $O R$ odds ratio, $P F O$ patent foramen ovale, TTH tensiontype headache

\begin{tabular}{lllll}
\hline & MHA $(n=100)$ & TTH $(n=100)$ & Control $(n=50)$ & $p$ value \\
\hline Age (mean \pm SD) & $17-57(34.6 \pm 9.3)$ & $15-60(33.8 \pm 10.4)$ & $17-59(32.1 \pm 10.0)$ & 0.352 \\
Sex & & & & \\
Female & 80 & 80 & 40 & 1 \\
Male & 20 & 20 & 10 & \\
\hline
\end{tabular}

\begin{tabular}{lllll}
\hline & TTH $(n=100)$ & MHA $(n=100)$ & OR (95\% CI) & $p$ value \\
\hline PFO & & & & \\
Negative $(n$ and \%) & $77(77 \%)$ & $50(50 \%)$ & $3.35(1.82-6.15)$ & $<0.001$ \\
Positive $(n$ and \%) & $23(23 \%)$ & $50(50 \%)$ & & \\
Large PFO/ASA & & & & \\
Negative $(n$ and \%) & $89(89 \%)$ & $65(65 \%)$ & & \\
Positive $(n$ and \%) & $11(11 \%)$ & $35(35 \%)$ & & \\
\hline
\end{tabular}


In addition, recent data indicate a close association between the presence of $\mathrm{CH}$ and atrial septal abnormalities including PFO [3-5], raising the possibility of a cause and effect relationship between these anomalies and different types of headache. Our findings, however, showed that there was no significant difference between the prevalence of PFO in our patients with TTH when compared with normal volunteers with no headache.

Furthermore, we found a significantly higher prevalence of PFO in migraineurs when compared with patients with tension headache $(p<0.001)$ and the normal subjects. This was also true for the collective presence of large PFOs and ASAs $(p<0.001)$.

Recent emerging data show an association between MHA, especially those with aura and PFO [11]. Migraine with aura is more strongly associated with larger shunts. In their study of 400 patients, Wilmshurst et al. [12] found that $53 \%$ of patients with large shunts had migraine with aura, whereas only 25 and $4 \%$ of those with medium and small shunts had these findings, respectively.

Our findings also showed a strong association between the presence of migraine headache and the presence of large PFOs/ASAs. These anomalies were present in $35 \%$ of MHA patients, while they were seen in only $11 \%$ of subjects with TTH $(p<0.001)$ and $10 \%$ of normal subjects. This finding is important because ASAs have been implicated as a potential source of embolic strokes and their association with PFO could further increase the risk of recurrent stroke in young adults [13].

\section{Study limitations}

There are some limitations in our study that include the higher frequency of females in our three groups, the relatively small number of the headache-free normal subjects and inclusion of patients with only one type of TTH, i.e. chronic tension headache.

Of course a large-scale study including all types of tension headache and a larger number of headache-free normal participants of all ages could solve some of these limitations.

Measurement of type-II error was not preplanned in present study and this is of course a shortcoming of this study because assuming the percent difference of PFO frequency between the tension headache patients and the control group to be $7 \%(23-16 \%)$ with an $\alpha=0.05$, the post hoc type-II error of the study would be 0.82 .

\section{Conclusion}

The presence of atrial septal abnormalities does not have a significant association with chronic tension headache and their detection can neither be used for treatment plans nor for clarification of pathogenetic mechanisms of the disease when compared with migraineurs.

Conflict of interest None.

\section{References}

1. Schwedt TJ, Demaerschalk BM, Dodick DW (2008) Patent foramen ovale and migraine: a quantitative systematic review. Cephalalgia 28:531-540

2. Overell JR, Bone J, Lees KR (2000) Interatrial septal abnormalities and stroke: a meta-analysis of case-control studies. Neurology 55:1172-1179

3. Finocchi C, Del Sette M, Angeli S, Rizzi D, Gandolfo C (2004) Cluster headache and right-to-left shunt on contrast transcranial Doppler: a case-control study. Neurology 63:1309-1310

4. Morelli N, Gori S, Cafforio G, Gallerini S, Baldacci F, Orlandi G, Murri L (2005) Prevalence of right-to-left shunt in patients with cluster headache. J Headache Pain 6:244-246

5. Dalla Volta G, Guindani M, Zavarise P, Griffini S, Pezzini A, Padovani A (2005) Prevalence of patent foramen ovale in a large series of patients with migraine with aura, migraine without aura and cluster headache, and relationship with clinical phenotype. J Headache Pain 6:328-330

6. Bigal ME, Lipton RB (2005) Tension-type headache: classification and diagnosis. Curr Pain Headache Rep 9:423-429

7. Headache Classification Committee of the International Headache Society (2004) The international classification of headache disorders, 2nd edn. Cephalalgia 24(Suppl 1):1-160

8. Lamy C, Giannesini C, Zuber M, Arquizan C, Meder JF, Trystram D, Coste J, Mas JL (2002) Clinical and imaging findings in cryptogenic stroke patients with and without patent foramen ovale. The PFO-ASA study. Stroke 33:706-711

9. Lipton RB, Scher AI, Kolodner K, Liberman J, Steiner TJ, Stewart WF (2002) Migraine in the United States: epidemiology and patterns of health care use. Neurology 58:885-894

10. Hagen PT, Scholz DG, Edwards WD (1984) Incidence and size of patent foramen ovale during the first 10 decades of life: an autopsy study of 965 normal hearts. Mayo Clin Proc 59:17-20

11. Sztajzel R, Genoud D, Roth S, Mermillod B, Le Floch-Rohr J (2002) Patent foramen ovale, a possible cause of symptomatic migraine: a study of 74 patients with acute ischemic stroke. Cerebrovasc Dis 13:102-106

12. Wilmshurst PT, Nightingle S, Walsh KP, Morrison WL (2005) Clopidogrel reduces migraine with aura after transcatheter closure of persistent foramen ovale and atrial septal defects. Heart 91:1173-1175

13. Berthet K, Lavergne T, Cohen A, Guize L, Bousser MG, Le Heuzey JY (2000) Significant association of atrial vulnerability with atrial septal abnormalities in young patients with atrial septal abnormalities in young patients with ischemic stroke of unknown cause. Stroke 31:398-403 